\title{
BASE Jumping Injuries Presenting to Emergency Departments in the United States: an Assessment of Morbidity, Emergency Department, and Inpatient Costs
}

\author{
Joseph D. Forrester, MD, MSc; Kirbi Yelorda, MD; Lakshika Tennakoon, MPhil, MSc; David A. Spain, MD; \\ Kristan Staudenmayer, MD, MS
}

Department of Surgery, Stanford University, Stanford, CA

\begin{abstract}
Background-BASE (building, antenna, span, earth) jumping involves jumping from fixed objects with specialized parachutes. BASE jumping is associated with less aerodynamic control and flight stability than skydiving because of the lower altitude of jumps. Injuries and fatalities are often attributed to bad landings and object collision.

Methods-We performed a retrospective analysis of the 2010-2014 National Emergency Department Sample database, a nationally representative sample of all visits to US emergency departments (EDs). BASE jumping-associated injuries were identified using International Classification of Diseases, Ninth Revision, Clinical Modification codes [E004.0]. Outcomes evaluated included morbidity, mortality, inpatient admission, and costs. Adjusted analyses accounting for survey methodology were performed.

Results-After weighting, 1790 BASE-associated ED presentations were identified with $358 \pm 28$ injuries annually. A total of 1313 patients (73\%) were aged 18 to $44 \mathrm{y}$, and $1277(71 \%)$ were male. Nine hundred seventy-six (55\%) multiple body system injuries and $677(38 \%)$ isolated extremity injuries were reported. There were $1588(89 \%)$ patients discharged home from the ED; only 144 (7\%) were admitted as inpatients. On multivariate logistic regression, only anatomic site of injury was associated with inpatient admission (odds ratio=0.6, $P<0.001,95 \%$ CI $0.5-0.8$ ). Including ED and inpatient costs, BASE injuries cost the US healthcare system approximately $\$ 1.7$ million annually. No deaths were identified within the limitations of the survey design.
\end{abstract}

Conclusions-Although deemed one of the most dangerous extreme sports, many patients with BASE injuries surviving to arrival at definitive medical care do not require inpatient admission.

Keywords: injury, healthcare costs, sports, trauma, wilderness, recreation

\section{Background}

BASE (building, antenna, span, earth) jumping involves jumping from fixed objects with specialized parachutes at altitudes much lower than that of skydiving. ${ }^{1}$ The term was coined by Carl Boenish, an iconic BASE jumper and filmmaker who filmed the first jumps from El Capitan in Yosemite Valley in 1978. ${ }^{1}$ In the ensuing decades, the sport has increased in popularity, but there is no formal governing body or verifiable number of participants.

Corresponding author: Joseph D. Forrester, MD, MSc, Department of Surgery, Stanford University, 300 Pasteur Drive, H3591, Stanford, CA 94305; e-mail: jdf1@ @stanford.edu.

Submitted for publication November 2018.

Accepted for publication February 2019.
Estimated injury rates are 5 to 10 times that of skydiving. Increased potential for injury is attributed to less aerodynamic control and decreased flight stability because of the lower altitude of jumps., ${ }^{2,3}$ In skydiving, jumps are typically made from approximately $3000 \mathrm{~m}$, and parachutes are deployed around $600 \mathrm{~m} .^{3}$ Many BASE jumps start around 300 to $600 \mathrm{~m}$, sometimes as low as $120 \mathrm{~m}$, and parachutes must be deployed within seconds. ${ }^{2,3}$ Uncontrolled parachute opening that results in collision with another object, termed object strike, is the most common cause of injury and death in BASE jumping. ${ }^{1,4}$ However, injuries incurred during landings can also result in injury or death. ${ }^{2}$ Wingsuit flying, a specialized form of BASE jumping, may be particularly dangerous and deadly. ${ }^{5}$ We examined the epidemiology, morbidity, 
mortality, and emergency department (ED) and inpatient healthcare cost of BASE jumping injury in the United States.

\section{Methods}

We performed a retrospective analysis of the 2010-2014 National Emergency Department Sample (NEDS) database to describe the epidemiology of BASE jumping injury among persons presenting to EDs in the United States. ${ }^{6}$ NEDS is the largest all-payer ED database in the United States, capturing both ED encounters that result in discharge or transfer and encounters that result in admission. Publically available, NEDS is constructed from the Healthcare Cost and Utilization Project State Emergency Department Databases and the State Inpatients Databases. When analyzed, NEDS is weighted to provide national estimates of ED care; the stratified sample of US hospital-based EDs represents approximately $20 \%$ of all US ED visits. The number of hospitals included in the sample ranged from 945 to 961 and reported 149,704,876 (667,095,064 after weighting) ED encounters over the 5-y time period.

We included all adult ED encounters with the diagnosis code corresponding to BASE jumping injury (International Classification of Disease, 9th Revision, Clinical Modification [ICD-9CM] E004.2). An extended definition of adult was used (age $>16$ y) to reflect the demographics of those recreationally participating in BASE jumping. No distinction was made between injuries obtained in different types of BASE jumping because this distinction is not made by the ICD coding. ${ }^{5}$ Variables assessed included age, sex, region, payer status, income quartile, site of injury, injury severity score, ${ }^{7,8}$ length of stay, discharge destination, and cost of ED encounter or hospitalization. Costs were derived from reported charges converted to costs using Healthcare Cost and Utilization Project cost-to-charge ratios. Injury characteristics were further evaluated with the Statabased ICD Programs for Injury Categorization module. The 2010-2014 time period was chosen because these databases were both available and had sufficient coding similarity to allow combinatorial analysis.

Outcomes of interest included mortality, inpatient admission, and cost. All statistical analyses used the NEDS sampling strata and discharge weights to produce nationally weighted patient-level estimates and 95\% CIs that account for clustering of patients among hospitals. ${ }^{10}$ Weighted values are reported unless otherwise specified. Univariate and multivariate analysis was performed. Multivariate logistic regression was performed compensating for survey methodology. An a priori $P$ value of $<0.05$ was considered significant. We used Stata 12.0
(StataCorp, College Station, TX) for all statistical analyses. The study was classified as exempt by the institutional review board because it contained no identifiable data.

\section{Results}

After weighting, 1790 ED visits were identified, corresponding to $358 \pm 28$ BASE-associated injuries presenting annually (Table 1). This corresponds to 3 BASEassociated injuries per 1 million patient visits. A total of 1313 patients $(73 \%)$ were aged 18 to $44 \mathrm{y}$, and 1277 (71\%) were male. The greatest frequency $(n=631,35 \%)$ of patients presented in the West region, followed by the South $(n=454,30 \%)$, the Midwest $(n=415,23 \%)$, and the Northeast $(n=199,11 \%)$. The majority $(n=1318,74 \%)$ of injuries occurred from April to October. Only 204 (11\%) patients presented to rural hospitals. Most ( $\mathrm{n}=793,44 \%)$ patients had private insurance, but patients from all 4 income quartiles were represented. Among patient presenting to the ED, 976 (55\%) had multiple body system injuries, and 677 (38\%) had isolated extremity injuries. There were 1588 (89\%) patients discharged home from the ED; only $144(7 \%)$ were admitted as inpatients. No deaths were reported.

Demographic data of patients admitted after BASE injury mirrored that of those patients who presented to the ED. On univariate analysis, admitted patients had more frequent isolated head, chest, and isolated extremity injuries than those discharged from the ED; they also had higher injury severity scores $(P<0.001)$. Six of the 10 most common inpatient procedures involved orthopedic stabilization or surgery; the most common procedure was surgical stabilization of tibia/fibula fractures. Median length of inpatient stay was $1 \mathrm{~d}$ (range $<1$ to $27 \mathrm{~d}$ ). Less than $1 \%$ of all inpatients required non-surgery-related intubation. On multivariate logistic regression, only the type of injury was associated with inpatient admission (odds ratio $=0.6, P<0.001,95 \%$ CI $0.5-0.8$ ). The total cost of patients with BASE jumping-associated injury presenting to the ED, including inpatient costs, was $\$ 8,396,933$ (95\% CI $\$ 2,239,587-\$ 14,500,000)$ for the 1790 visits over the 5 -year time period.

\section{Discussion}

BASE jumping understandably draws considerable interest along a spectrum of individuals, from participants to observers to policymakers. Commonly considered an "extreme sport," BASE jumping is becoming increasingly recognizable by the lay public. ${ }^{2,3}$ Because of the inherent risk associated with jumping from these structures, BASE jumping sites may be tightly regulated, such as in the US National Parks. ${ }^{111}$ Yet there is little definitive epidemiologic data describing the morbidity 
Table 1. Epidemiology of patients presenting to the emergency department after BASE injury-United States, 2010-2014

\begin{tabular}{|c|c|c|}
\hline Characteristics & $\begin{array}{l}B A S E(\%) \text { injuries presenting } \\
\text { to } E D, \text { weighted }\end{array}$ & $\begin{array}{l}\text { BASE (\%) injuries requiring } \\
\text { admission, weighted }\end{array}$ \\
\hline No. & 1790 & 144 \\
\hline \multicolumn{3}{|l|}{ Age (y) } \\
\hline Less than 18 & $222(12)$ & $27(18)$ \\
\hline $18-44$ & $1313(73)$ & $107(75)$ \\
\hline $45-64$ & $229(13)$ & $10(7)$ \\
\hline $65-74$ & $14(1)$ & - \\
\hline $75-84$ & $11(1)$ & - \\
\hline Male sex & $1277(71)$ & $114(79)$ \\
\hline \multicolumn{3}{|l|}{ Region } \\
\hline Northeast & $199(11)$ & $10(7)$ \\
\hline Midwest & $415(23)$ & $40(28)$ \\
\hline South & $545(30)$ & $20(14)$ \\
\hline West & $631(35)$ & $75(52)$ \\
\hline \multicolumn{3}{|l|}{ Payment data } \\
\hline Medicare & $65(4)$ & $5(4)$ \\
\hline Medicaid & $378(21)$ & $33(23)$ \\
\hline Private insurance & $793(44)$ & $61(42)$ \\
\hline Self-pay & $429(24)$ & $36(25)$ \\
\hline No charge & $3(0)$ & - \\
\hline Other & $123(7)$ & $9(6)$ \\
\hline \multicolumn{3}{|l|}{ Household income compared to ZIP code } \\
\hline $0-25$ th & $391(22)$ & $39(27)$ \\
\hline 26th -50 th & $145(28)$ & $41(29)$ \\
\hline $51 \mathrm{st}-75 \mathrm{th}$ & $468(26)$ & $39(27)$ \\
\hline 76th-100th & $422(24)$ & $24(17)$ \\
\hline \multicolumn{3}{|l|}{ Injury burden } \\
\hline Isolated head/neck & $82(5)$ & $19(13)$ \\
\hline Isolated chest & $36(2)$ & $22(16)$ \\
\hline Isolated abdomen & $20(1)$ & $4(3)$ \\
\hline Isolated extremity & $677(38)$ & $72(50)$ \\
\hline Multiple body regions & $976(55)$ & 27 (19) \\
\hline Injury severity score $>15$ & $15(1)$ & $15(10)$ \\
\hline Median length of stay (range), $d$ & & $1(<1$ to 27$)$ \\
\hline \multicolumn{3}{|l|}{ Discharge destination } \\
\hline Died in ED & - & - \\
\hline Home & $1588(89)$ & $119(83)$ \\
\hline Admitted as inpatient & $144(8)$ & $\mathrm{n} / \mathrm{a}$ \\
\hline Short-term rehabilitation & $21(1)$ & $5(3)$ \\
\hline Skilled nursing facility & $9(0)$ & $15(10)$ \\
\hline Home health & $4(0)$ & $5(4)$ \\
\hline Against medical advice & $23(1)$ & - \\
\hline Total cost for ED encounters (95\% CI) & $\$ 2,925,589(\$ 2,137,681-\$ 3,713,497)$ & - \\
\hline Total cost for hospital encounters $(95 \% \mathrm{CI})$ & - & $\$ 8,396,933(\$ 2,239,587-\$ 14,500,000)$ \\
\hline
\end{tabular}

BASE, building, antenna, span, earth; ED, emergency department; n/a, not applicable.

and mortality associated with participating in this sport. To place the risk of BASE jumping in context, we evaluated the NEDS to examine the epidemiology of BASE injuries in the United States.

Over the 5-y time period assessed in our study, among injured BASE jumpers who arrived at definitive medical care alive, only $7 \%$ of injuries resulted in admission, and no deaths were reported. In 2012, the severe injury rate, defined as an injury requiring that the participant seek medical care, was estimated to be 2 per 1000 BASE jumps. ${ }^{2}$ This estimate was created based on self-reported injuries obtained through a cross-sectional survey. Based 
on this estimated severe injury rate and the estimated number of injuries seen in US EDs, approximately 895,000 BASE jumps took place across the United States from 2010-2014.

The anatomic pattern of injury seen among BASEassociated injury in NEDS is similar to previously published studies. Among BASE jumpers who self-reported severe injury from 2006-2010, extremities $(\mathrm{n}=24$, $61 \%)$, back/spine $(\mathrm{n}=8,20 \%)$, chest wall $(\mathrm{n}=7,18 \%)$, and head injuries $(n=5,13 \%)$ were commonly reported. ${ }^{2}$ This injury pattern of BASE jumpers is similar to that of skydivers, understandable given the considerable risk of injury to the lower extremities during landing. ${ }^{12}$ However, the reported risk of lower extremity injury is considerably higher for BASE jumpers compared to skydivers; fracture risk from BASE jumping is estimated to be 12 times that of skydiving. ${ }^{2}$ The preponderance of lower extremity bony injury is consistent with the frequency of tibia/fibular orthopedic fixation required among admitted patients in our series.

No fatalities were identified in the NEDS analysis. Over the same time period, 24 deaths while BASE jumping or wingsuit jumping in the United States were reported by the BASE jumping community. ${ }^{13}$ Similarly, in 2008, a descriptive analysis of 106 fatal BASE jumps estimated the fatality risk to be 1 death per 60 participants per year, and reports from Norway estimate 1.1 fatality per 2500 jumps. ${ }^{1,4}$ The absence of fatalities detected through NEDs can be interpreted in several ways. It is possible that no fatalities were detected secondary to sampling error inherent in survey data. Another possibility is that fatal events are intentionally or unintentionally underreported or not reported, although this is less likely when evaluating patients once they have made contact with the medical system, such as with NEDS. A third explanation is that BASE jumpers generally are so severely injured that they die on impact or before transport to definitive care or they have relatively minor injuries. BASE injury data from Norway support the latter hypothesis; among the 9 deaths from 1995-2005, 8 died immediately, and 1 died before contact with rescuers. ${ }^{4}$ Similar fatality patterns have been observed among injured rock climbers. ${ }^{14,15}$

There is scant data describing the healthcare cost of extreme sports, BASE jumping in particular. ${ }^{16}$ Our study found that BASE jumping-related injuries cost the US healthcare system $\$ 1.68$ million each year for ED and inpatient costs alone. This is likely a marked underestimate as our study only captures patients presenting to EDs; does not account for deaths that occur at the time of injury or during prehospital transport; and does not include physician costs, outpatient costs, rehabilitation costs, or costs attributable to work lost. Rescue of injured jumpers, or body recovery costs, from austere sites is expensive and may require helicopter support and highangle rescue. ${ }^{3,4}$ As a result, the overall cost of BASErelated injuries to the US healthcare system based on our data alone is an underestimate of the true total healthcare cost. Similar estimates are not available from other countries to our knowledge and represent an opportunity for further research.

There are several limitations to this study. First, only BASE jumpers injured in the United States were evaluated. It is possible that injury patterns experienced in the United States may differ from that in other countries, limiting extrapolation. Second, the experience levels of the injured BASE jumpers were not available, preventing any assessment of the impact of experience on injury pattern or rates. Third, there may be misclassification bias associated with using ICD-9 codes; the validity of the codes depends on the quality of coders at each participating institution. In the ICD-9 code, recreational BASE jumping is not specified, and it is possible people unintentionally falling from structures could be misreported as BASE jumping. This misclassification could affect the number of recreational BASE-related injuries, and this could vary by hospital region. Fourth, NEDS reports event-level data but does not have unique identifiers for any given individual. An individual BASE jumper who experiences multiple injuries over the course of the year would be counted as multiple jumpers. This may lead to overestimation of the number of injured BASE jumpers, particularly given that $43 \%$ of BASE jumpers in historical series reported repeat injuries. ${ }^{2}$ Fifth, because of the variably legal status of BASE jumping, it is possible that patients or patients' family and friends may not accurately report the circumstances surrounding an injury. This misrepresentation could lead to falsely low rates of injury. Finally, although NEDS is the most comprehensive survey of EDs in the United States, it may not accurately capture EDs in close proximity to popular BASE jumping areas. This could lead to overestimation or underestimation of BASE-related injury.

\section{Conclusions}

BASE jumping is an inherently risky sport. Injured jumpers are commonly male, in their third to fifth decade of life. Many injured BASE jumpers presenting to US EDs do not require admission. No patients died upon reaching definitive medical care among the NEDS survey sample, although deaths in healthcare facilities not submitting data to NEDS would not have been captured. Anatomic injury patterns are consistent with described mechanisms of injury including object strike and difficult landings. ED and inpatient healthcare costs of 
BASE jumping-associated injury are considerable and may increase as BASE jumping increases in popularity.

Author Contributions: Writing (JDF, KY, KS); data collection (LT); data analysis (JDF, KY, LT, KS); data interpretation (JDF, KY, KS).

Financial/Material Support: None.

Disclosures: None.

\section{References}

1. Westman A, Rosen M, Berggren P, Bjornstig U. Parachuting from fixed objects: descriptive study of 106 fatal events in BASE jumping 1981-2006. Br J Sports Med. 2008;42 (6):431-6.

2. Mei-Dan O, Carmont MR, Monasterio E. The epidemiology of severe and catastrophic injuries in BASE jumping. Clin J Sport Med. 2012;22(3):262-7.

3. Soreide K. The epidemiology of injury in bungee jumping, BASE jumping, and skydiving. Med Sport Sci. 2012;58: 112-29.

4. Soreide K, Ellingsen CL, Knutson V. How dangerous is BASE jumping? An analysis of adverse events in 20,850 jumps from the Kjerag Massif, Norway. J Trauma. 2007;62 (5):1113-7.

5. Mei-Dan O, Monasterio E, Carmont M, Westman A. Fatalities in wingsuit BASE jumping. Wilderness Environ Med. 2013;24(4):321-7.

6. HCUP Nationwide Emergency Department Sample (NEDS). Healthcare Cost and Utilization Project (HCUP). 2010-2014. Rockville, MD: Agency for Healthcare Research and Quality. Available at: www.hcup-us.ahrq. gov/nedsoverview.jsp. Accessed January 17, 2019.
7. Boyd CR, Tolson MA, Copes WS. Evaluating trauma care: the TRISS method. Trauma Score and the Injury Severity Score. J Trauma. 1987;27(4):370-8.

8. Glance LG, Osler TM, Mukamel DB, Meredith W, Dick AW. Expert consensus vs empirical estimation of injury severity: effect on quality measurement in trauma. Arch Surg. 2009; 144(4):326-32. discussion 332.

9. Clark ED. ICDPIC Version 3.0; ICD-9-CM Trauma within STATA version 11.2.

10. Healthcare Cost. Utilization Project. HCUP Online Tutorial Series. Rockville, MD: Agency for Healthcare Research and Quality; 2019. Available at: https://www. hcup-us.ahrq.gov/tech_assist/tutorials.jsp. Accessed 15 January 2019.

11. National Park Service. Management policies. Washington, DC: US Department of the Interior; 2006:103.

12. Lee CT, Williams P, Hadden WA. Parachuting for charity: is it worth the money? A 5-year audit of parachute injuries in Tayside and the cost to the NHS. Injury. 1999;30(4):283-7.

13. BLiNC Magazine. Fatality statistics. 2018. Available at: http://www.blincmagazine.com/forum/wiki_index.php? title=Fatality_Statistics. Accessed 27 June 2018.

14. Bowie WS, Hunt TK, Allen Jr HA. Rock-climbing injuries in Yosemite National Park. West J Med. 1988;149(2):172-7.

15. Forrester JD, Tran K, Tennakoon L, Staudenmayer K. Climbing-related injury among adults in the United States: 5-year analysis of the National Emergency Department Sample. Wilderness Environ Med. 2018;29 (4):425-30.

16. Caine DJ. The epidemiology of injury in adventure and extreme sports. Med Sport Sci. 2012;58:1-16. 